NASA Technical Memorandum 104345

AIAA-91- 2822

\title{
The Dynamic Effects of Internal Robots on Space Station Freedom
}

Jeffrey H. Miller

Sverdrup Technology, Inc.

Lewis Research Center Group

Brook Park, Ohio

and

Charles Lawrence and Douglas A. Rohn

National Aeronautics and Space Administration

Lewis Research Center

Cleveland, Ohio

Prepared for the

Guidance, Navigation and Control Conference

sponsored by the American Institute of Aeronautics and Astronautics

New Orleans, Louisiana, August 12-14, 1991

\section{N/SA}





\title{
THE DYNAMIC EFFECTS OF INTERNAL ROBOTS ON SPACE STATION FREEDOM
}

\author{
Jeffrey H. Miller \\ Sverdrup Technology, Inc. \\ Lewis Research Center Group \\ Brook Park, Ohio 44142 \\ Charles Lawrence and Douglas A. Rohn \\ National Aeronautics and Space Administration \\ Lewis Research Center \\ Cleveland, Ohio 44135
}

\section{Abstract}

Many of the planned experiments for space station Freedom will require acceleration levels to be no greater than microgravity $\left(10^{-6} \mathrm{~g}\right)$ levels for long periods of time. Studies have demonstrated that without adequate control, routine operations may cause disturbances which are large enough to affect on-board experiments. One way to both minimize disturbances and make Freedom more autonomous is to utilize robots instead of astronauts for some operations. The present study addresses the feasibility of using robots for microgravity manipulation. Two methods for minimizing the dynamic disturbances resulting from the robot motions are evaluated. The first method is to use a robot with kinematic redundancy (redundant links). The second method involves the use of a vibration isolation device between the robot and the space station laboratory module. The results from these methods will be presented along with simulations of robots without disturbance control.

\section{Introduction}

\section{Disturbances}

space station Freedom and other space based platforms would benefit greatly by having on-board, or internal, robots to perform delicate, time consuming, or repetitive tagks. Many of the experiments which have been proposed for study on the space station require disturbance levels to be lesg than one million times smaller than the gravitational acceleration seen on Earth $\left(10^{-6} \mathrm{~g}\right.$ or $1 \mu \mathrm{g})$. Dynamic disturbances from adjacent experiments, operating equipment, station maneuvers, and even astronaut motions, must be controlled in order to insure that these levels are maintained. ${ }^{1}$ Also, there is a need to decrease the reaction forces and moments which will be created by the apparatus used in conducting the experiments. One way to minimize the disturbances, and also make Freedom more autonomous, is to utilize robots instead of astronauts for some operations. Since properly designed robots move in a more controlled manner than humans, robots will be better suited to perform the acceleration sensitive functions which will be required for microgravity experimentation. Furthermore, robots may be used for tagks which are labor intensive or difficult for humans to perform.

Typical astronaut motions, such as exercising and pushing off of a wall may be large enough to cause disturbances which exceed the $1 \mu \mathrm{g}$ limit. This has been shown both experimentally on the space shuttle and $5 k y l a b^{1,2}$ and through computer simulations. The disturbances from the astronauts come both from the nonprecise nature of human motions and the inability of humans to accurately sense when they create small disturbances. Robots may move more smoothly than humans, and therefore cause less disturbances to the space platform.

Another aspect that favors the use of robots is the great demand on the astronauts" time. They are involved in 
the general operations and piloting of the space platform, and cannot therefore devote all of their time to the laboratory experiments. The time demands on the astronauts will drastically limit the number of experiments that can be carried out. Robots can help with the workload and can conduct experiments when the astronauts are not on the space platform, thereby increasing the amount of research that can be performed.

Robots will only be useful, however, if they do not contribute disturbances of their own to the space platform. This paper will show that robots are capable of maintaining reduced acceleration levels. Robots may even reduce the disturbances that will result from the required motion of some components of the experiment while the experiment is being carried out. The primary concern now is with the effects of the robot on the space platform, although eventually the effects of the platform on the robot will also need to be considered. Figure 1 is a diagram of the system being considered.

This study is similar to Harman, et $a 1 .{ }^{3}$ The present study uses examples which are more representative of typical robot tasks whereas Harman addressed extremely large payloads. This study also looks at the use of redundant robots and a vibration isolation device while the Harman study was limited to nonredundant robots.

In Largen, et al. ${ }^{2}$ the feasibility of lsolating the space station treadmill is investigated. This work is similar to the present in that the objective of both investigations is to assess the possibilitles of dynamic isolation for maintaining the microgravity environment. In Ref. 2 it is concluded that isolation may be practically accomplished with minimal treadmill displacements and required clearances. However, it should be noted that the treadmill weight had to be increased from 65 to $1000 \mathrm{lb}$ to lower the treadmill resonances and accomplish the desired level of isolation. Also, the treadmill was not located directly in the microgravity laboratory as is the case with the present robot study. Furthermore, the proposed isolation scheme in Ref. 2 requires a modification of the current microgravity acceleration requirements because the resulting treadmill acceleration levels actually exceed the current requirements.

Methods of Isolation

The present study addresses the feasibility of using robots for microgravity manipulation. Two methods for minimizing the dynamic disturbances resulting from robot motions are evaluated. The first method involves the use of a robot with kinematic redundancy. These kinematically redundant robots have more degrees of freedom than are necessary for carrying out their tasks. For example, if the task requires the robot to move an object in a plane then 2 degrees of freedom are required. Any additional degrees of freedom are the redundant ones. By having these extra degrees of freedom, the robot can move parts of the robot in a direction opposite to that of the end-effector and payload. This cancels some, or all, of the disturbance effects of the robot motion. For this type of robot there are an infinite number of joint trajectories which will accomplish the desired endeffector path. By optimally selecting the joint trajectory which minimizes the reaction forces at the base of the robot, it is possible to reduce the resulting dynamic disturbances.

The second method for reducing the dynamic effects involves the use of a vibration isolation device (VID) between the robot and the space station laboratory module. This device can help isolate disturbances from the robot onto the space station and from the space station onto the robot and experiment. A VID is typically composed of springs, dampers, and masses and acts like a filter to reduce the transmission of disturbances above a threshold frequency. VIDs with fixed physical parameters are considered as well as VIDs with tuneable, adjustable parameters. 


\section{Computer simulation}

A computer simulation of space Station Freedom is used to analyze the effects of disturbances. A NASTRAN finite element model of an early version of the space station has been modified for use in this study. This model, which has a mass of $141000 \mathrm{~kg}(312000 \mathrm{lb})$, is shown in Fig. 2. The results obtained are from a dynamic modal transient analysis using the first 11 fundamental modes of the station coupled to the robot and vibration isolation system. The results of a convergence study on the reduced model shows that the modal model performs successfully using only the first 11 modes. This greatly reduces the computational effort required while not significantly changing the results. Component substructuring was used for the cases in which the VID is placed between the robot and the space station to allow for the complete solution of the VID while maintaining the reduced model for the space station itself. When the space station is built it will most likely not be identical to the present computer model. However, the results will still be qualitatively correct and the conclusions drawn will still be relevant. In fact, any space based platform on which a microgravity environment is needed will have similar disturbance minimization problems.

Figures 3 and 4 show the result of a computer simulation of an astronaut moving about on the space station. Figure 3 shows the force used for this simulation. A $22 \mathrm{~N}$ (5 1b) force was applied in one direction for 1 sec and was then applied for 1 sec in the opposite direction. This is equivalent to an astronaut pushing off of a wall in the laboratory module and then coming to rest against a wall on the other side. Figure 4 shows the magnitude of the resulting acceleration, which can reach $18 \mu \mathrm{g}$. The force is applied within the United States Laboratory Module, and the accelerations are measured at this same location. This is where the microgravity laboratories will be gtationed. Figure 5 shows the simulated result of an astronaut exercis- ing on a treadmill. The astronaut has a mass of $81 \mathrm{~kg}(180 \mathrm{lb})$ and jogs with an amplitude of $1.2 \mathrm{~cm}(3 \mathrm{in.})$. This far exceeds the microgravity disturbance Iimit.

Programs which calculate robot joint trajectories as well as forces and moments at the base of the robot are used to determine the input data for the $f$ inite element space station model. These codes were written under grant at Carnegie Mellon University and Case western Reserve University. (See Refs. 4 to 7 for more information.) In addition to the robot dynamics codes a separate optimization program was developed to determine the physical parameters for the VID.

The robot models used for this study were designed with physical specifications which are believed to be realistic given the proposed tasks they will have to perform. The robots used for the dynamic simulation are shown in Figs. 6 to 8. All of the joints are revolute. Robots with differing numbers of degree of freedom are used to show the effect of having differing degrees of redundancy. The robots are similar in all other aspects. They have the same length, mass, and workspace. They are also required to follow the same end-effector trajectories. Therefore any differences in the base reactions are due entirely to the different number of redundancies.

For a given robot model, the trajectory planning programs choose the optimal joint angles based on the desired starting and ending positions for the robot payload. The program also minimizes the forces and moments at the base of the robot for the chosen trajectory. The resulting base forces and moments then are used with the finite element space station model. The modal transient analysis is performed and the resulting acceleration levels at the laboratory module are recorded.

It is interesting to note that while for both terrestrial and space robots it is important to limit the disturbances on 
the payload at the robot end-effector, with space-based robots the base forces also are important since the base may move, and forces can be transmitted through the base. For terrestrial robots the base is usually assumed to have a sufficiently large mass that it cannot move, and forces transmitted through the base are therefore often of no concern.

\section{Results and Discussion}

Nonredundant Robot

If there are robots on the space station, one of their tasks will certainly involve moving relatively small objects around the laboratory module. These objects could be associated with the actual experiments such as test tubes or specimen holders, or may be related to general housekeeping chores. For tasks of this nature most of the disturbances will come from the robot motion itself. The small end-effector payloads will not significantly affect the disturbances. A specific task was chosen for this study which requires a $24 \mathrm{~kg}(53 \mathrm{lb})$ robot to move an object $0.3 \mathrm{~m}(11.8 \mathrm{in}$.$) in 2 \mathrm{sec}$ along a prescribed trajectory. It is assumed that the robot is fixed at the base. For the robot to complete this task successfully it must not disturb other experiments which might be in progress.

The effect of the nonredundant robot (Fig. 6) will be determined first. This robot was used with the robot dynamics programs and the space station model. The robot is two dimensional (planar) while the space station model is three dimensional. This lessens the computational requirements and makes it simpler to compare results while maintaining their significance. The forces and moments generated by the nonredundant robot can be seen in Fig. 9. Figure 10 shows the magnitude of the resulting accelerations of the laboratory module. This was obtained by taking the square root of the sum of the squares of the accelerations in each of the three coordinate directions. The maximum regulting acceleration is $4.7 \mu \mathrm{g}$. This level exceeds the allowable microgravity limit and is therefore unacceptable. This is, however, probably no worse than the disturbances which would arise from an astronaut performing the same task.

\section{Redundant Robots}

The effect of using redundant robots will now be compared to the previous results for the nonredundant robot. As was mentioned earlier, the only difference between the redundant and nonredundant robots is the number of links in each robot. The end-effector trajectories are the same. The redundant robots, shown in Figs. 7 and 8 , have one and two redundancies respectively. The forces and moments generated by these robots can be seen in Figs. 11 and 12 . Figures 13 and 14 show the resulting accelerations of the laboratory module. The robot with one redundancy causes a maximum acceleration of $3.0 \mu \mathrm{g}$ while the robot with two redundancies leads to a maximum acceleration of $0.95 \mu \mathrm{g}$. Figure 15 shows a comparison of the nonredundant robot with the redundant robots. As the number of redundancies increases the disturbances get smaller. The dashed line on Fig. 15 is the allowable $1 \mu \mathrm{g}$ disturbance limit. With two redundancies the disturbances do not exceed this limit. Different tasks, particularly those requiring motion in three-dimensional space, may require more redundancies to maintain the $1 \mu \mathrm{g}$ limit. obviously the computational effort increases as the number of redundancies increases. The extra effort is worth taking, however, if it is necessary in order to guarantee the successful completion of a disturbance sensitive experiment.

\section{Vibration Isolation Device}

The second method of reducing disturbances involves the use of a vibration isolation device. The signiflcance of using robots with various amounts of redundancy has already been addressed. Therefore, the focus will now be on changing the VID parameters while the robot model remains the same for all of 
the test cases. A nonredundant robot with a mass of $27.3 \mathrm{~kg}(60.2 \mathrm{lb})$, including a payload of $9 \mathrm{~kg}(20 \mathrm{lb})$, was used (Fig. 16). It was commanded to move the payload $0.53 \mathrm{~m}(21 \mathrm{in.})$ in $1 \mathrm{sec}$. This is believed to be representative of a typical robot task such as setting up experimental apparatug. The maximum disturbance level caused by this robot without a VID is $150 \mu \mathrm{g}$. These disturbance will exceed the allowable acceleration level.

The simplest method for dynamic isolation of the robot from the space station Freedom structure is to utilize a passive isolation system. The isolation system, which consists of an inertial mass, linear spring and damper, would be situated between the robot and the space station structure. The structural characteristics of the isolator would be determined by examining the expected magnitude and frequency content of the robot base reactions. Since the isolator is passive (i.e., constant mass, stiffness, and damping) its characteristics could only be optimal for a specific robot motion, and would be less than optimal for the complete range of motions which the robot may undergo.

To assess the effectiveness of a passive isolation system, the nonredundant robot shown in Fig. 16 is placed on an isolation system, and the resulting displacements and acceleration levels aboard the space station are examined. The robot motion is identical to the previous examples. This motion results in base forces and moments which are then input into the NASTRAN finite element model of the entire space station including the isolation system. Finally, a modal transient analysis is performed, for different isolator characteristic frequencies, to determine the resulting displacements and acceleration levels at key locations aboard the space station.

In order to model the VID/space station system, the substructuring capabilities of NASTRAN are utilized. The space station and the VID are each defined separately; the station is modeled modally and the VID is modeled with physical mass, stiffness, and damping elements. This modeling approach is used because it is computationally efficient and because the VID is relatively stiff in comparison to the station. since the VID is stiff, a conventional model transient analysis would be ineffective because an excessive number of modes would be required in order to capture the behavior of the VID. By substructuring the system, the flexible components (i.e., space station) can be modeled modally and the stiff component (i.e., VID) can be modeled with physical elements.

Figure 17 shows the resulting accelerations and displacements as a function of isolator frequency. The accelerations were taken at the base of the isolator where it would be attached to the space station, while the displacements are from the base of the robot. The displacements at the base of the robot are of interest because as the isolator becomes more effective (i.e., less transmitted load),the displacements increase and may become excessively large. The accelerations at the base of the isolator are important because they determine the amount of disturbance which would be transmitted into the space station infrastructure.

Three sets of results, each using a different isolator weight, were generated for this figure. While all three weights cause different displacements, they all result in the same acceleration levels. This is because the transmitted acceleration is dependent only on the isolator frequency, not the isolator mass or stiffness.

In order to determine what acceleration will result from a particular mass and displacement, first locate the point of interest on one of the displacement curves. Then move vertically from the displacement curve to the acceleration curve. The resulting acceleration is represented by the point where the vertical line intersects the acceleration curve. 
As shown in the figure, as the isolator frequency increases, its effectiveness decreases and the transmitted acceleration becomes quite large. For very stiff, high frequency isolatorg, the isolator is completely ineffective and acceleration depends only on the robot base forces and the mass of the space station. For very soft or low frequency isolators, the isolator is very effective in reducing acceleration levels, but the resulting displacement at the robot base become prohibitively large. For example, a $22.7 \mathrm{~kg}$ (50 lb) passive isolator designed to satisfy a $1 \mu \mathrm{g}$ requirement would need to have a displacement of nearly $50 \mathrm{~cm}(20 \mathrm{in.})$. Displacements of this magnitude could present not only serious clearance problems, but could also severely effect the robot positioning capabilities. Obviously, a heavier isolator may be used to obtain an effective isolation level with less displacement, but there would be an increase in complexity and cost.

For low frequency isolation (less than $0.01 \mathrm{~Hz}$ ), all three curves level of $f$ and the displacements reach a maximum displacement for each of the three isolator weights considered. This is because the "soft" isolator begins to behave as a freely floating mass dependent only on the isolator mass, not the stiffnegs. For higher frequency isolation (greater than $1.0 \mathrm{~Hz}$ ), the displacements are very small, but the corresponding isolation effectiveness is minimal.

\section{Tuneable Isolation}

In the previous section a passive isolation device was shown to improve the disturbances resulting from robot motions. However, robot motions can cover a wide range of frequencies. The passive isolator with fixed properties will only be effective when the disturbance frequency is above the critical frequency of the VID, and in other ranges the 1solator can even amplify the disturbances. Therefore, tuneable isolation may ultimately be required to accommodate the wide range of disturbances expected from a robot. With tuneable isolation the damping and stiffness properties can be adjusted as disturbance changes. since we already have shown that a robot with an isolation device helps reduce disturbances we will look only at the difference between tuneable and fixed isolation for a given robot. The robot behaves similarly on the either device, and any improvements of having a robot on a fixed VID can be safely extended to a robot on a tuneable VID.

The tuneable isolation system can be effective in situations where the robot base reactions are known a priori. The tuneable system could be used to optimally control the isolator stiffness and damping by minimizing a weighted cost function containing the isolator displacements and transmitted reactions. The cost function would be integrated over a period of time that is at least as long as it takes the robot to perform its trajectory. The advantage of the tuneable isolator is that it does not require any feedback loop. It is therefore the easiest adaptive system to implement. The disadvantage of the tuneable isolator is that the robot base reactions must be known and the associated isolator parameters computed before the robot trajectory is initiated.

The tuneable isolator may be designed by minimizing the cost function:

$$
\operatorname{Min} J=\int\left\{\mathbf{u}^{2}+\alpha \mathbf{R}^{2}\right\} d t
$$

where $\mathbf{u}$ are the six displacements at the isolator mass, $\mathbf{R}$ are the reactions transmitted through the isolator to the space station, and $\alpha$ is a weighting parameter.

The IMSL routine DUMINF, ${ }^{8}$ which employs the quasi-Newton method, is used for minimizing the above cost function. The required displacements and reactions are computed from the robot base reactions and numerical integration. For the integration, it is assumed that the isolator base, where it attaches to the 
isolator base, where it attaches to the space station, is fixed. This assumption reduces the isolator to a decoupled, 6-degree-of-freedom system thus greatly simplifying the numerical integration. The fixed base assumption is reasonable considering the relatively small mass of the isolator in comparison to the space station mass.

The isolator stiffness is determined by assuming a continuous, third order polynomial function (i.e., stiffness $\left.=\mathrm{p}_{1}+\mathrm{p}_{2} * \mathrm{u}+\mathrm{p}_{3} * \mathrm{u}^{2}+\mathrm{p}_{4}^{*} \mathrm{u}^{3}\right)$. This function was used so that the stiffness distribution would be relatively smooth and not change too abruptly in small time periods. The IMSL routine is used to compute the unknown parameters, $p_{1}$ through $\mathrm{p}_{4}$.

The overall procedure for designing the tuneable isolator is as follows. First, the robot base reactions are input into the cost function minimization routine where the optimal stiffness parameters are computed. The maximum isolator deflections are recorded for subsequent use. Next, the resulting transmitted isolator reactions resulting from the robot base reactions are applied to the space station model. Then a transient analysis is performed and the maximum resulting acceleration levels at the attachment point between the space station and the isolator is recorded. Finally, the procedure is rerun for different $a$ values and an isolator displacement versus space station acceleration curve is constructed.

The displacement versus acceleration curve for a $22.7 \mathrm{~kg}(50 \mathrm{lb})$ isolator mass (Fig. 18) shows the effect of varying $a$ in the cost function. For comparison purposes, the results from the fixed isolator also are shown in the figure. For stiff isolators with displacements less than $5 \mathrm{~cm}$ ( 2 in.), the resulting acceleration levels exceed $120 \mu \mathrm{g}$ and the fixed and tuneable isolators deliver comparable results. For flexible isolators having displacements greater than $40 \mathrm{~cm}$ (16 in.), the disturbance level is very small and fixed and tuneable isolators again produce equivalent results. In the range between 5 and $40 \mathrm{~cm}$ ( 2 and 6 in.), the fixed and tuneable isolators produce different results. In this range the tuneable isolator is more effective in minimizing the transmitted reactions. For example, at $12 \mathrm{~cm}$ (5 in.) of displacement the tuneable isolator is able to reduce the transmitted reactions so that the resulting acceleration aboard the space station is only $50 \mu \mathrm{g}$ while the fixed system is only able to reduce the level to slightly below $120 \mu \mathrm{g}$. Although the tuneable system is more effective in this region, displacements greater than $5 \mathrm{~cm}$ ( $2 \mathrm{in.}$ ) may not be tolerable in practice. For those cases in which the robot motion is known a priori the results obtained from using an isolation system with active feedback would not be any better than those for the tuneable system. The active system would adjust the physical parameters until they were the same as those used on the tuneable system.

\section{Heavy Payloads}

In all of the above results, the payloads are small and therefore most of the disturbance is caused by the actuating system (i.e., robot or astronaut) and not by the payload motion itself. It has been shown that these disturbances may be controlled to acceptable levels because a redundant robot can compensate for disturbances caused by its motion. It was not necessary in these cases to compensate for the payload. Large payloads greater than $50 \mathrm{~kg}$ (110 lb) can generate significant disturbances caused by the motion of the payload itself. For these situations it is necessary to compensate for the actuating system as well as for the payload. Redundant robots are able to do this. Similarly, a heavy payload cannot be rapidly moved by an astronaut without causing large disturbances because the astronaut does not have the ability to compensate for the payload. In this situation a reaction compensating device, such as a redundant robot with sufficient mass, must be used. 
One of the proposed microgravity experiments involves moving a furnace which has a mass of $2000 \mathrm{~kg}$. Harman, et al. ${ }^{3}$ looked at moving this furnace with a nonredundant robot. Their results showed that the experiment caused unacceptable disturbances. However, these disturbances are actually caused by the furnace itself and not by the robot. 'A redundant robot which is massive enough to compensate for the payload could be used to reduce the disturbance forces.

In order to demonstrate the effectiveness of redundancy for large payloads it will be shown that the disturbances at the base of a redundant robot with a heavy payload are smaller than the disturbance which would exist if the payload were moving by itself. The robot model and trajectory used in Fig. 8 will be used for the demonstration. The only differences are that the robot now has a large payload and link masses are much larger in order to compensate for this payload. The end link which will represent the payload has a mass of $100 \mathrm{~kg}$ (220 1b). The other links also each have a mass of $100 \mathrm{~kg}$. For the case in which the payload is moving by itself, the smallest possible peak acceleration required to move the payload must be determined in order to make a fair comparison to the robot-payload system. This acceleration occurs when the payload moves with a constant acceleration for the firgt half of the time period, and an equal but opposite deceleration for the second half. If the acceleration at any moment was smaller than this, then an acceleration which is larger than the constant acceleration value would be needed later on in order for the payload to reach the desired end point in the available time. The payload force is equal to this constant acceleration multiplied by the payload mass. Since the payload is initially at rest and also ends up at rest, the acceleration is determined from the equation:

$$
\frac{B}{2}=\frac{I}{2} a\left(\frac{t}{2}\right)^{2}
$$

where $\mathbf{a}$ is the total displacement, a is acceleration, and $t$ is the $t$ ime period in which the payload must complete its motion. In this example $s$ is $0.3 \mathrm{~m}$ and $t$ is 2 sec. Therefore $a$ is equal to $0.3 \mathrm{~m} / \mathrm{s}^{2}$. The force resulting from this acceleration is:

$$
F=100 \mathrm{~kg} \times 0.3 \mathrm{~m} / \mathrm{s}^{2}=30 \mathrm{~N}
$$

The disturbance forces for the robot-payload system are determined from the robot computer codes. The results are shown in Fig. 19(a). The force in both the $x$ and $Y$ directions are no greater than $18 \mathrm{~N}$ (4 $\mathrm{lb}$ ) at the base of the robot. The magnitude of the total force (i.e., the square root of the sum of the forces in $x$ and $y)$ is calculated in order to compare this result with the magnitude of the force resulting from the payload moving by itself. This comparison can be seen in Fig. 19 (b). The minimum possible peak force from the payload moving by itself is $30 \mathrm{~N}$ while the maximum force from the robot-payload system is only $18 \mathrm{~N}$. Although the trajectory optimization codes were used to minimize the base reactions, the actual design configuration of the robot was not optimized. It is only used to demonstrate that a redundant robot can compensate for the payload as well as for itself. The amount of disturbance reduction depends only on the number of redundancies and the design of the robot.

\section{Concluding Remarks}

Two methods of minimizing robot disturbances have been addressed. The first method, using kinematic redundancy, has been shown to reduce the disturbance levels for a typical robot motion from $4.7 \mu \mathrm{g}$ to less than $1 \mu \mathrm{g}$. The payload mass used in this example was small in comparison to the mass of the robot. This reduction in disturbance forces required the use of a robot with two redundant links.

The second method of reducing disturbances involved placing vibration isolation devices between the robot and the 
laboratory module. The effectiveness of these devices was shown to be dependent on their natural frequency. As the isolator frequency increases, their effectiveness decreases and the transmitted acceleration becomes quite large. For very soft or low frequency isolators, they are very effective in reducing acceleration levels, but the resulting' displacement at the robot base become prohibitively large.

A tunable VID was then compared to the passive VID with fixed parameters. For stiff isolators with displacements less than $5 \mathrm{~cm}$ ( $2 \mathrm{in}$ ) the fixed and tunable isolators deliver comparable results. For flexible isolators with displacements greater than $40 \mathrm{~cm}$ (16 in.) the disturbance level was very small and fixed and tunable isolators again produced equivalent results. In the range between 5 and $40 \mathrm{~cm}$ ( 2 and $16 \mathrm{in.}$ ), the tunable isolator was more effective in minimizing the transmitted reactions. Although the tunable system was more effective in this region, the resulting displacements might still be too large to be tolerated in practice.

It was also shown that redundant robots may allow heavy payload experiments to take place without disturbing the microgravity environment. These experiments would cause large disturbances without reaction compensation because the payload itself, not the actuating system, is causing the disturbances. This shows that the robot is able to compensate not only for itself but also for the disturbance caused by the payload motion.

Kinematic redundancy and vibration isolation can both be used to reduce disturbance forces. The only real difference is that with kinematic redundancy the reaction control is done within the robot while with the VID the reaction control is done separately from the robot. Both kinematic redundancy and vibration isolation are effective in reducing disturbance forces. The advantage of the VID is that it is simpler and is decoupled from the robot control problem. The advantage of kinematic redundancy is that there is no displacement at the robot base and no hardware is needed in addition to the robot itself. Kinematic redundancy and vibration isolation may also be used together. Every robot and every application are unique. Therefore, these methods of isolation would need to be compared to see which combination of isolation techniques would be most effective.

\section{References}

1. Dodd, W.R., Badgley, M.B., and Konkel, C.R., "User Needs, Benefits, and Integration of Robotic systems in a space station Laboratory," TBESSD-P601-89-191, Teledyne Brown Engineering, NASA CR-185150, Oct. 1989 .

2. Larsen, C.E. and Cao, T.T., "Feasibility of Treadmill Isolation for the Space station Freedom Microgravity Environment," 1989.

3. Harman, P.E. and Rohn, D.A., "The Impact of an IVA Robot on the space Station Microgravity Environment," AIAA Paper 89-0956, Jan. 1989.

4. Chung, C.L. and Desa, S., "A Global Approach For Using Kinematic Redundancy To Minimize Base Reactions of Manipulators Used In Microgravity Environments," presented at 1989 IEEE International Conference on Robotics and Automation. (Not published in Conference proceedings.) (NASA CR-186825, 1989.)

5. Chung, C.L., "A Unified Motion Planning Approach for Redundant and NonRedundant Manipulators with Actuator Constraints," Ph.D. Thesis, Dept. of Mechanical Engineering, Carnegie Mellon University, 1990. 
6. Quinn, R.D., Chen, J.L., and

Lawrence, C., "Redundant Manipulators for Momentum Compensation in a Micro-Gravity Environment, " AIAA Guidance, Navigation, and Control Conference, Part 2, AIAA, 1988, Pp. 581-587

7. Quinn, R.D. and Lawrence, C., "Robots for Manipulation in a Micro-Gravity

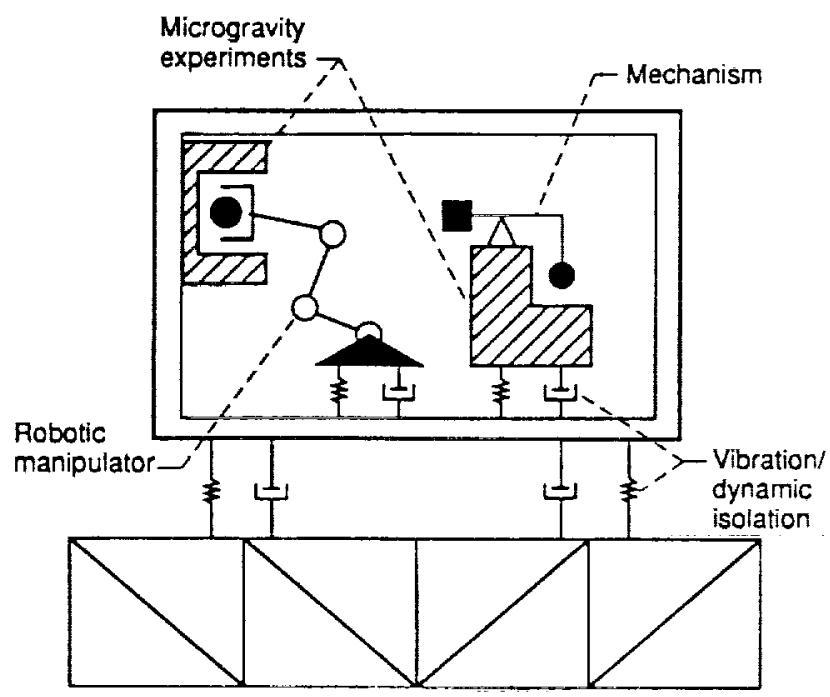

Space Station infrastructure

Figure 1.-Diagram of Robot-Space Station system.

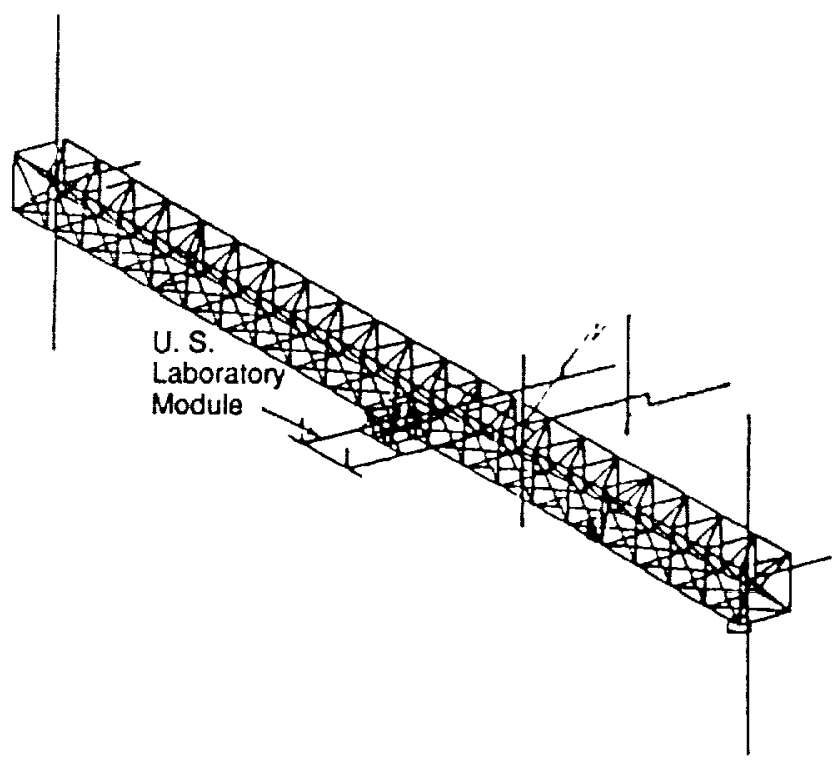

Figure 2.-Space station configuration.
Environment," Sixth Symposium on Dynamics and Control of Large Structures, L. Meirovitch, ed., Virginia Polytechnic Institute, 1987, pp. 515-528.

8. MATH/LIBRARY User's Manual, Version 1.0, IMSL Inc., Houston, 1987.

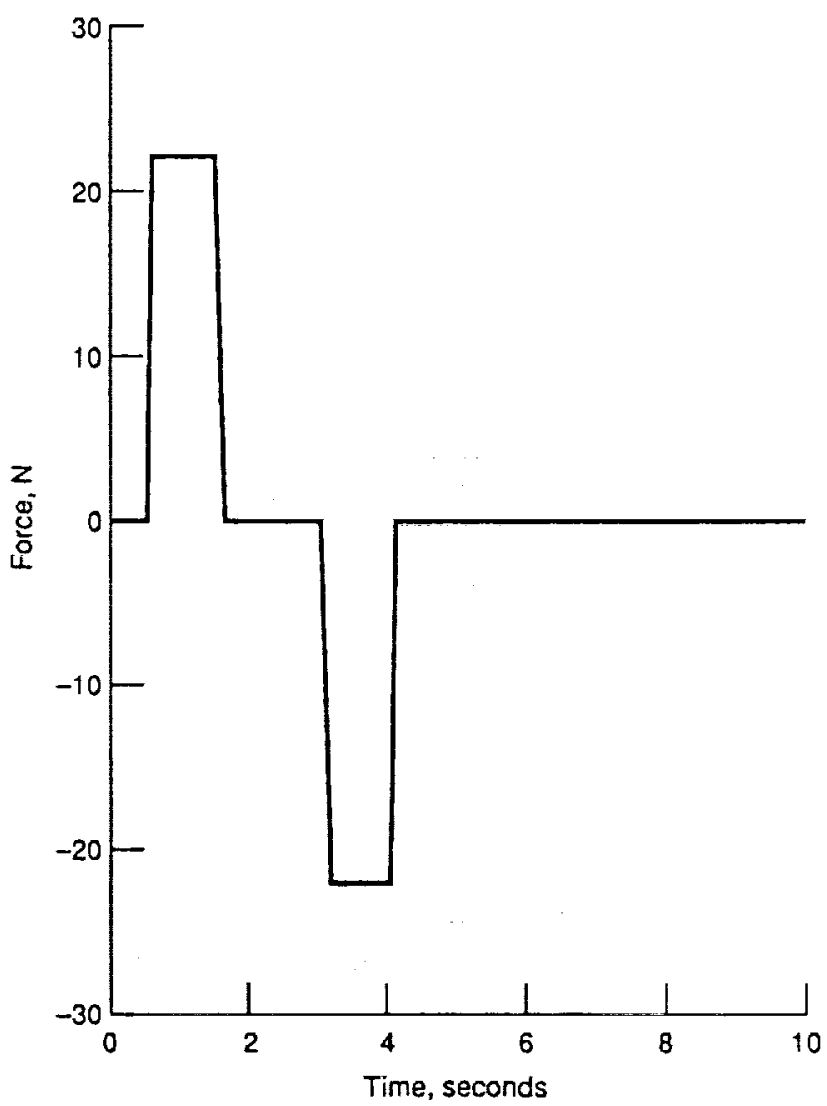

Figure 3.-Astronaut forces resulting from pushing off of a wall in the Space Station. 


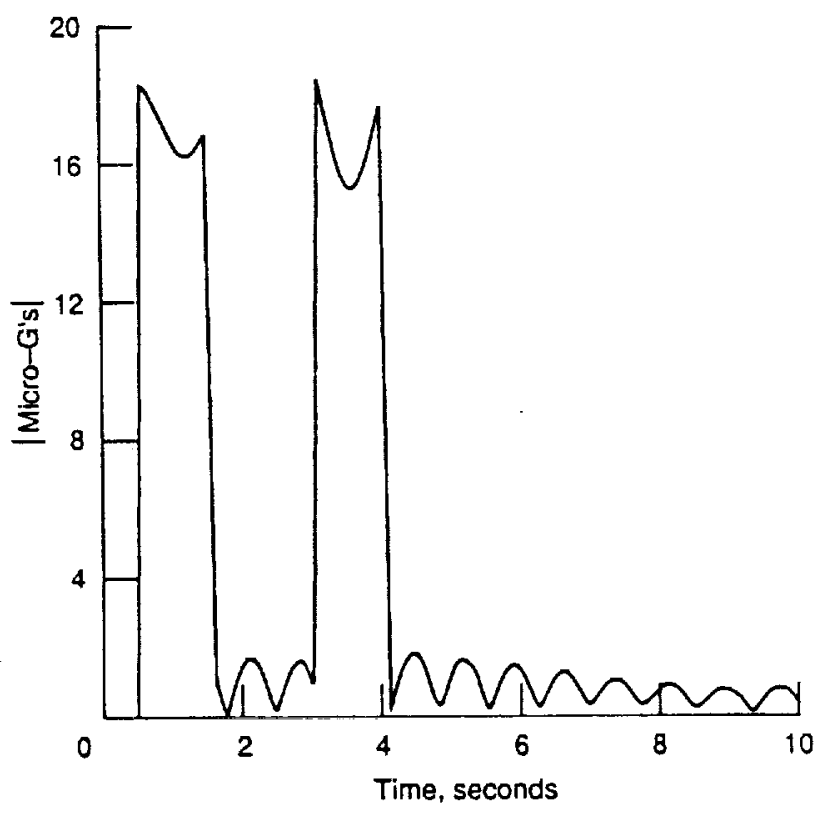

Figure 4.- Magnitude of the acceleration disturbance levels caused by an astronaut pushing off of a wall in the Space Station.

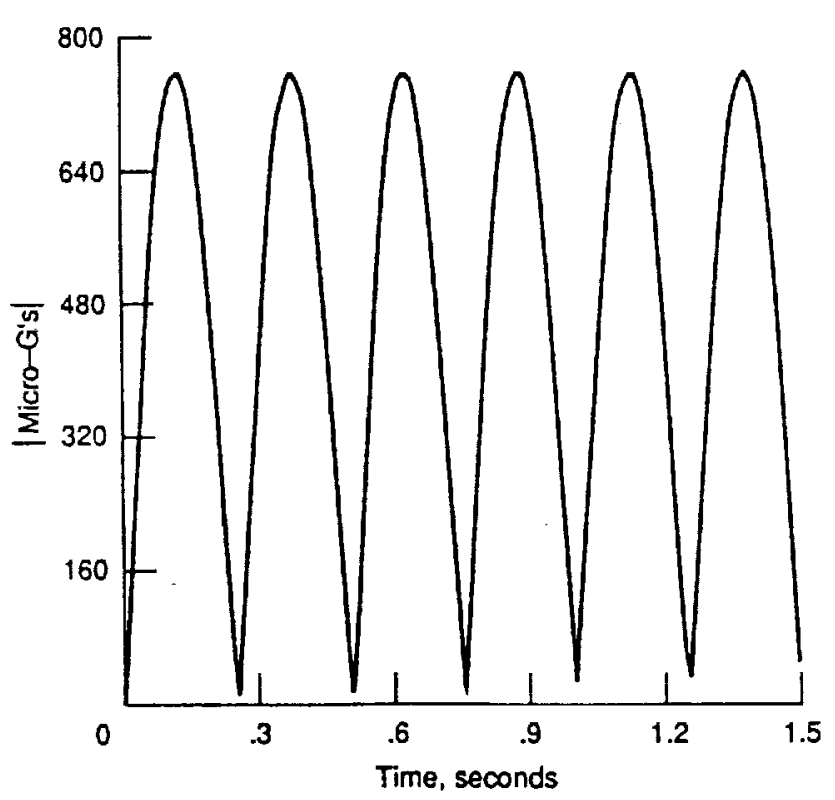

Figure 5.-Magnitude of the acceleration disturbance levels caused by an astronaut logging on a treadmill.

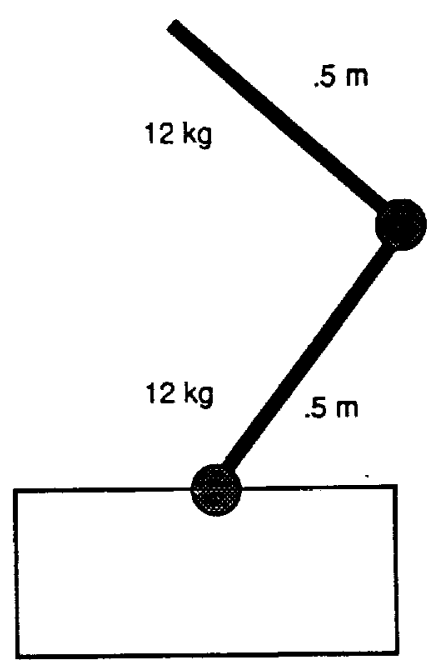

Figure 6.-Robot with no redundancies.

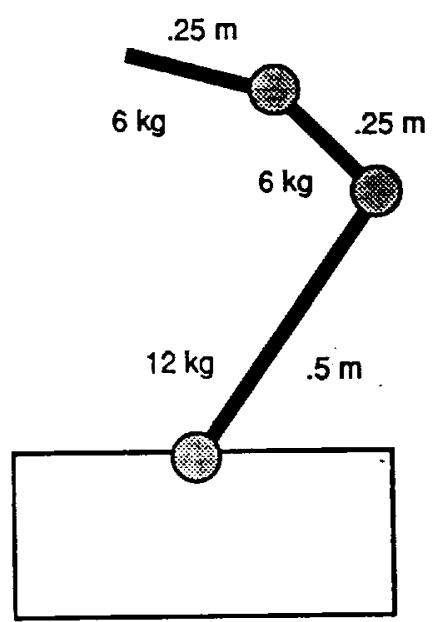

Figure 7.-Robot with one redundancy.

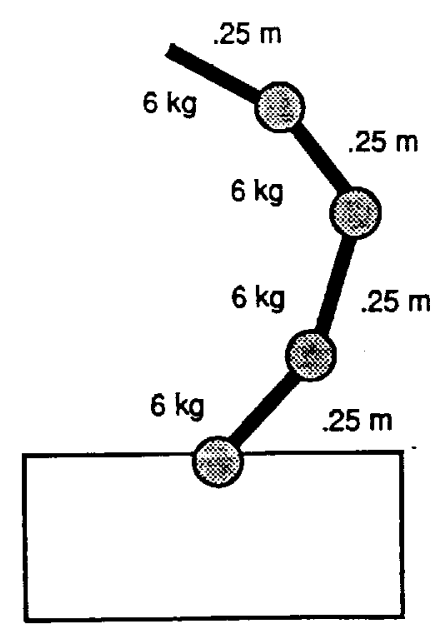

Figure 8.-Robot with two redundancies. 


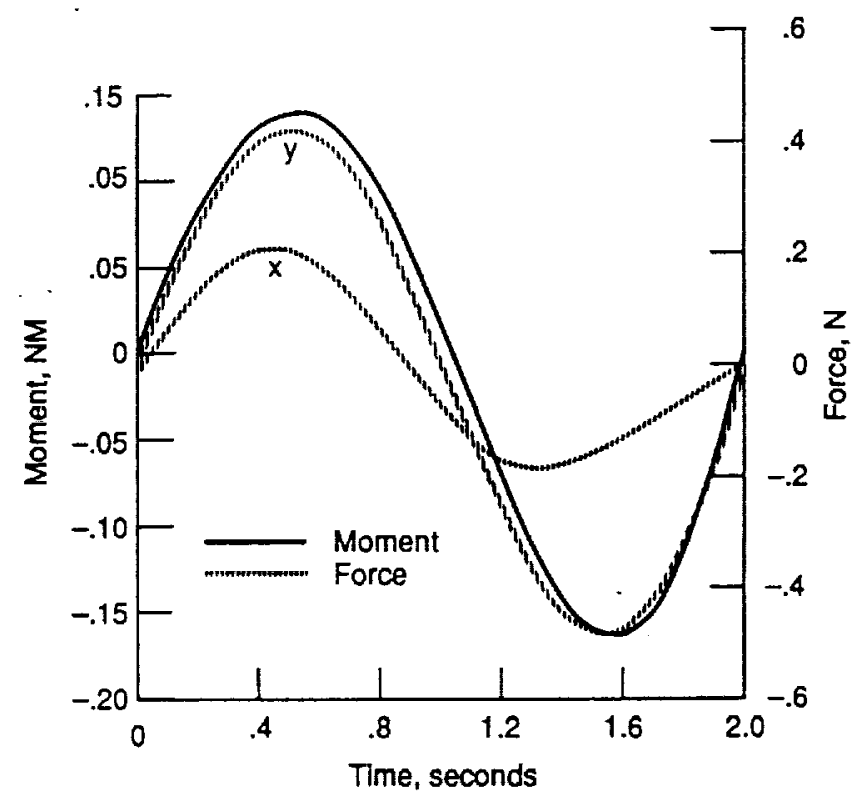

Figure 9.-Forces and moments generated by a non-redundant robot.

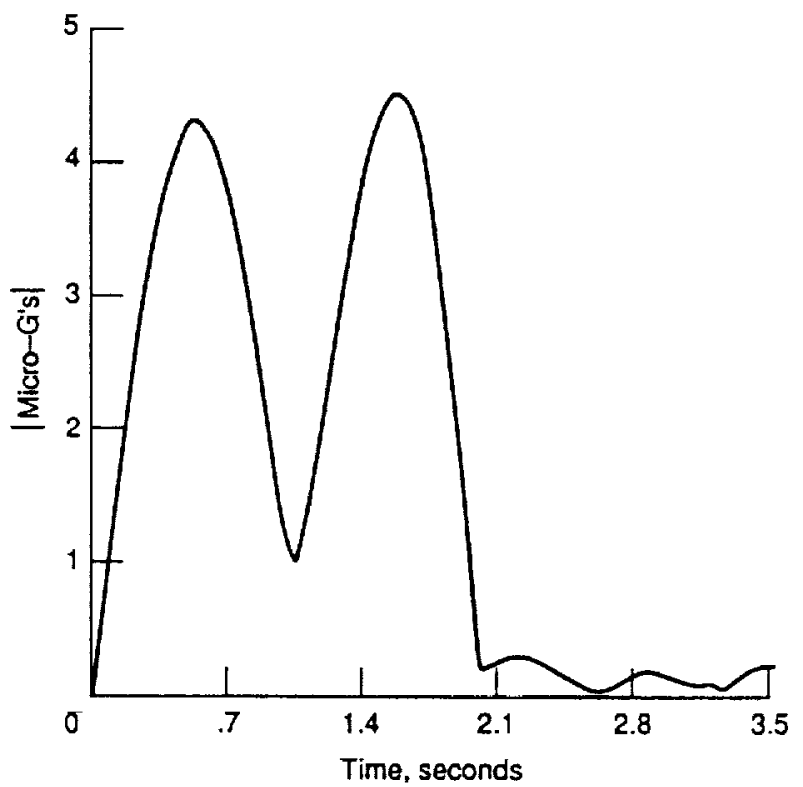

Figure 10.-Magnitude of the acceleration levels from the nonredundant robot.

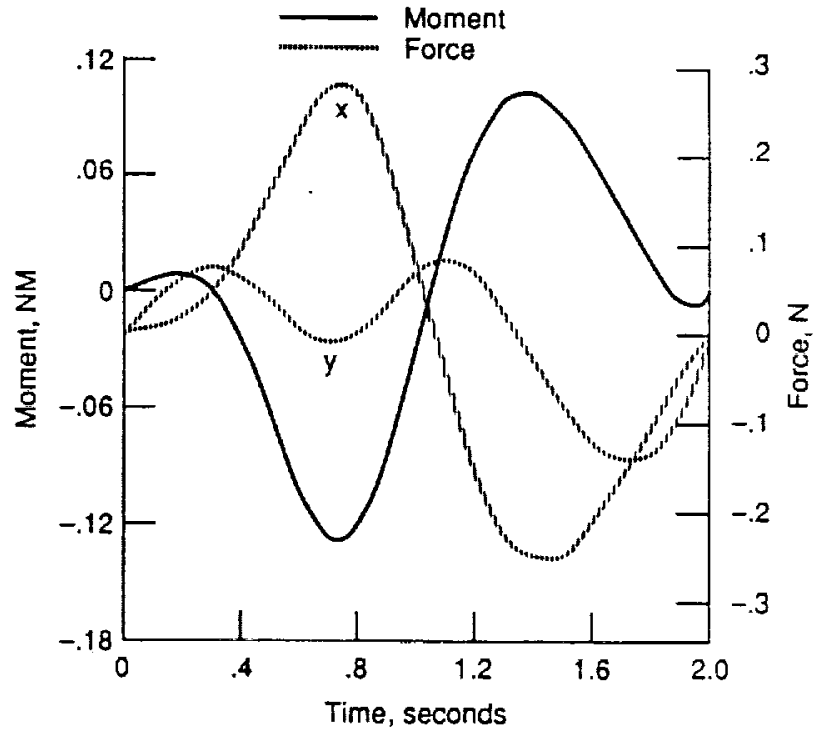

Figure 11.-Forces and moments generated by a robot with one redundancy.

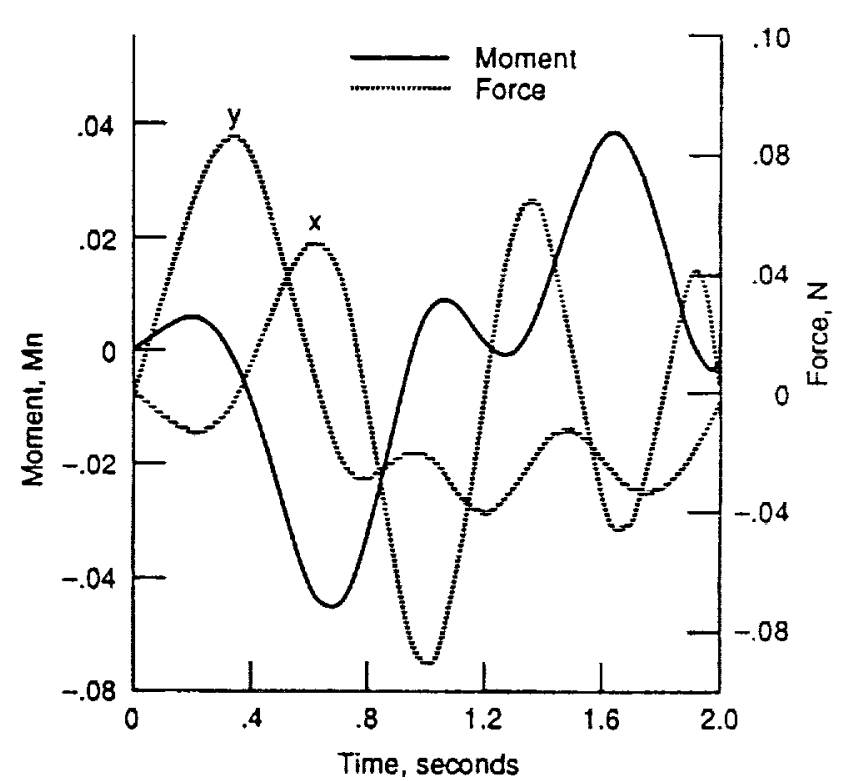

Figure 12.-Forces and moments generated by a robot with two redundancies. 


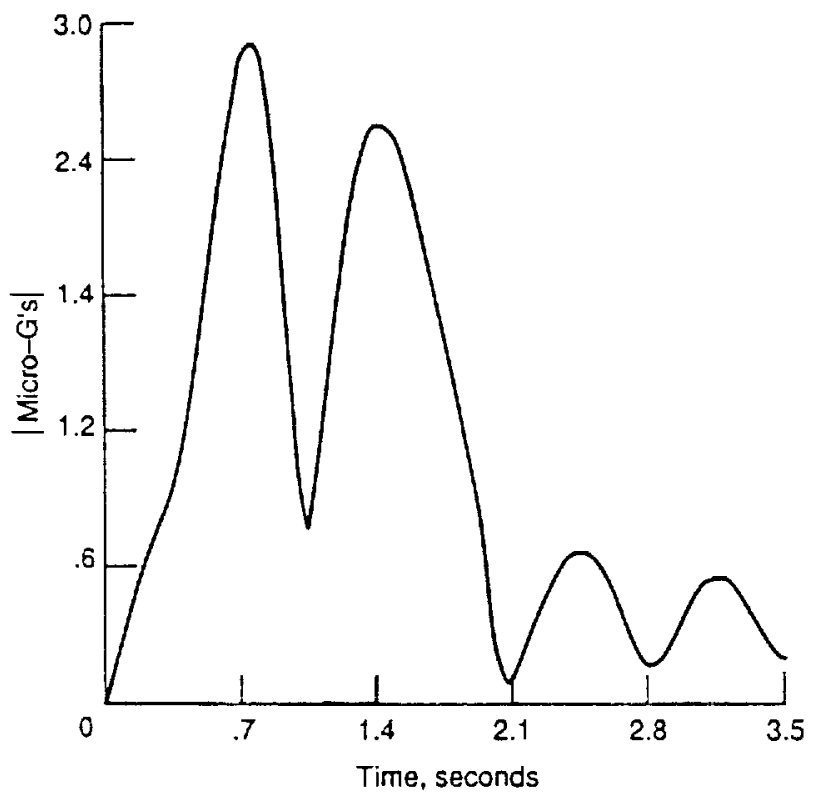

Figure 13.-Magnitude of the acceleration levels from the robot with one redundancy.

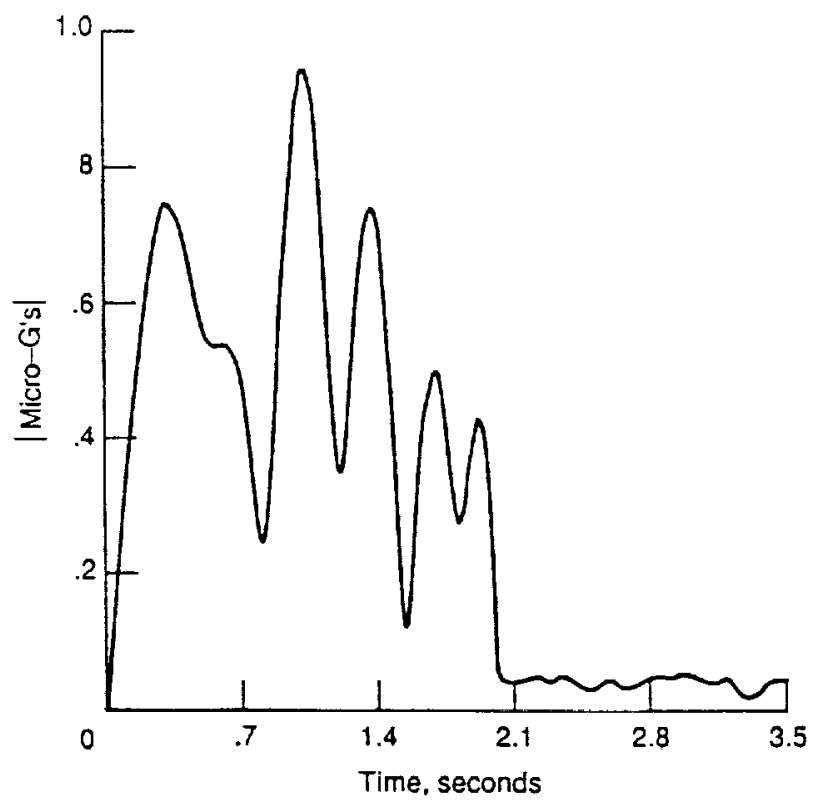

Figure 14.-Magnitude of the acceleration levels from the robot with two redundancies.

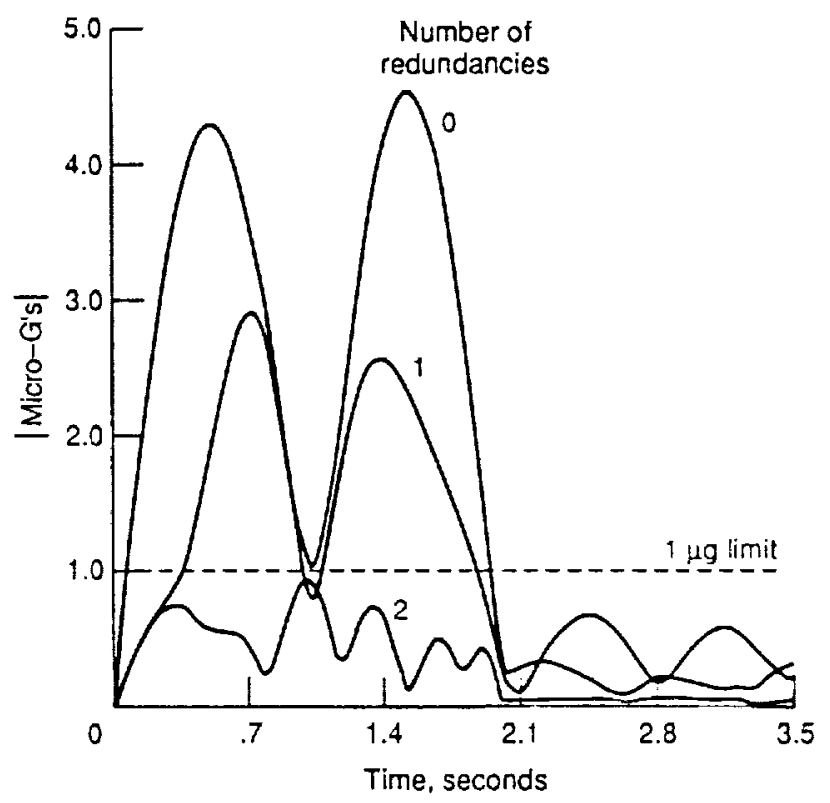

Figure 15.-Acceleration level comparison for 0.1 , and 2 redundancies.

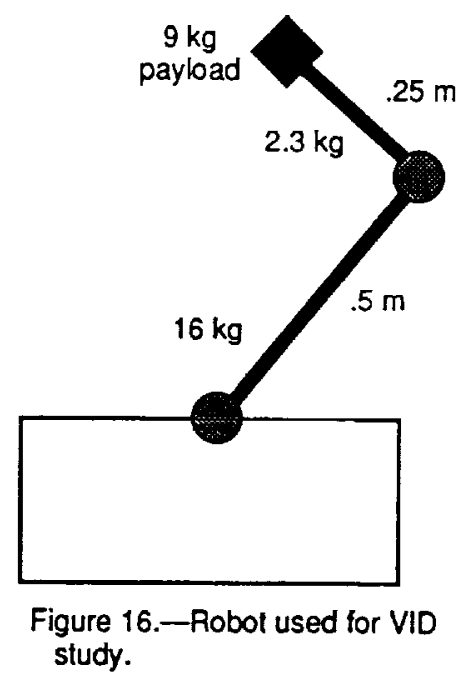



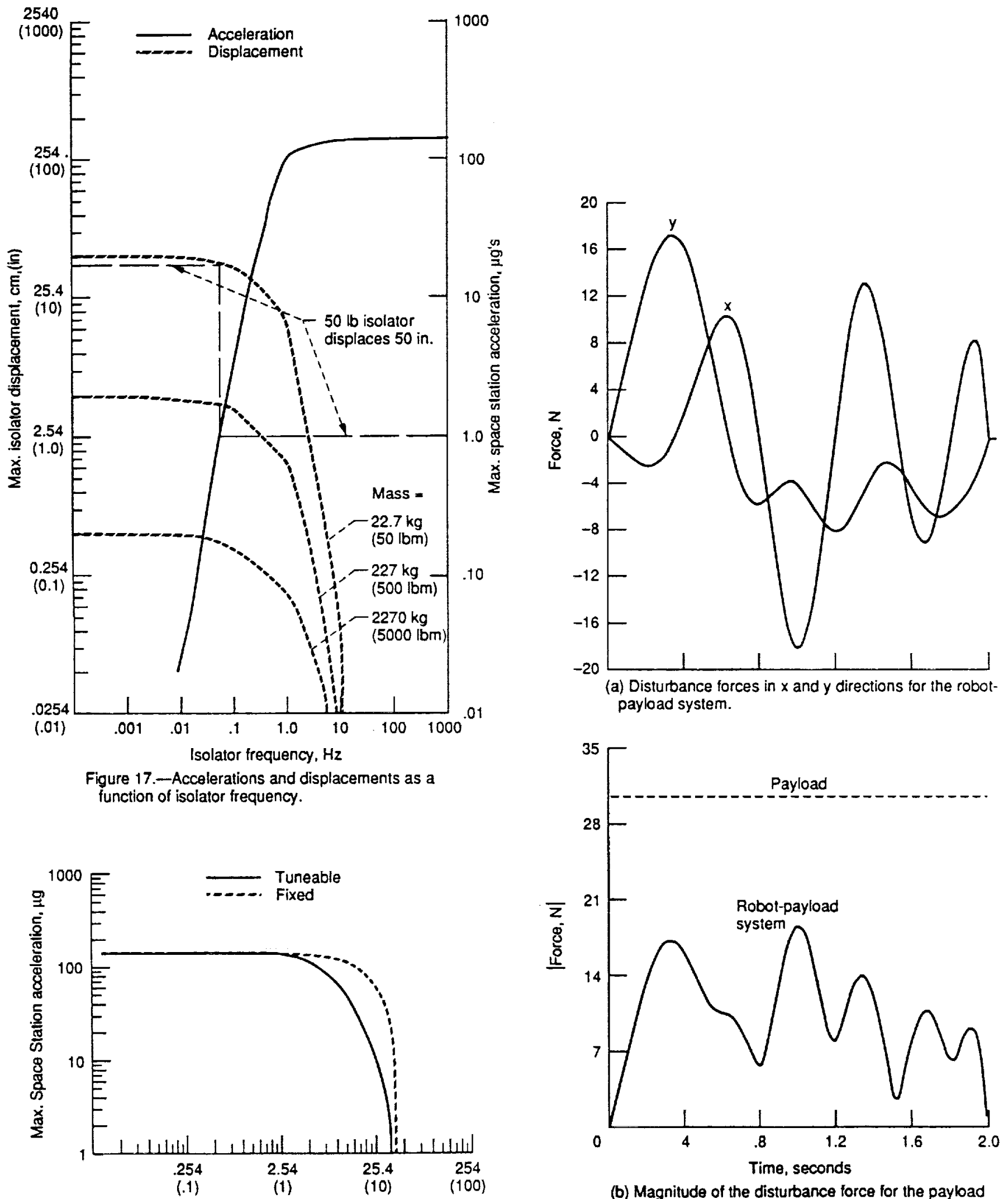

Max. displacement, cm, (in.)

Figure 18.-Displacement vs. acceleration curves for $50 \mathrm{lb}$ isolator mass. 


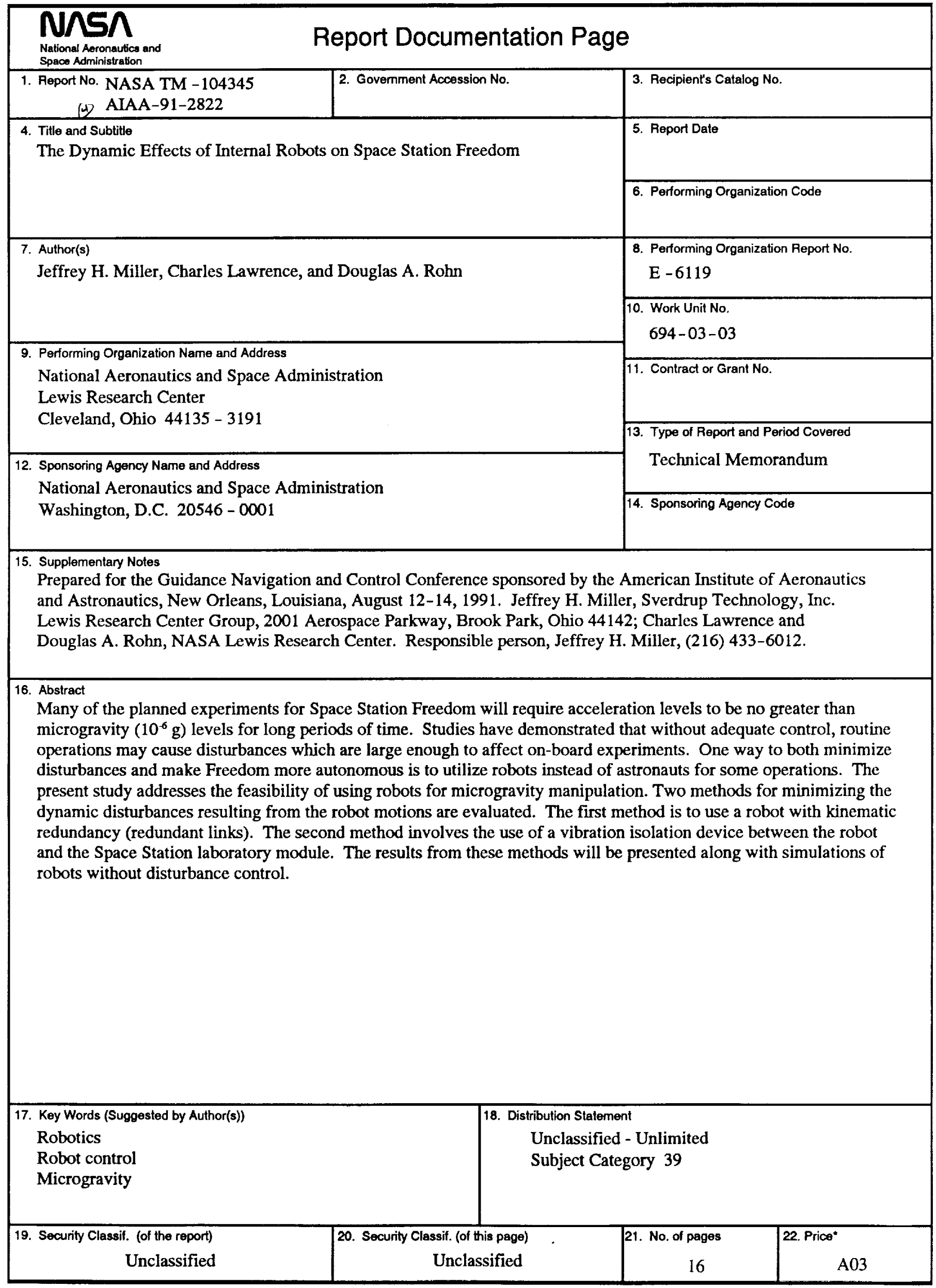

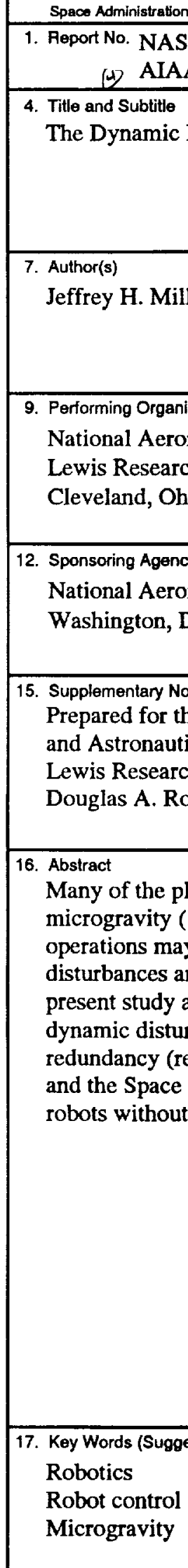


